\title{
SOX1 down-regulates $\beta$-catenin and reverses malignant phenotype in nasopharyngeal carcinoma
}

\author{
Zhong Guan ${ }^{1,2+}$, Jialiang Zhang ${ }^{1 \dagger}$, Jing Wang ${ }^{2 \dagger}$, Hefei Wang ${ }^{3 \dagger}$, Feimeng Zheng ${ }^{2,3}$, Jieren Peng ${ }^{1}$, Yaodong Xu',
} Min Yan', Bing Liu ${ }^{3}$, Bai Cui ${ }^{3}$, Ying Huang ${ }^{2}$ and Quentin Liư ${ }^{2,3^{*}}$

\begin{abstract}
Background: Aberrant activation of the Wnt/ $\beta$-catenin signaling pathway is an important factor in the development of nasopharyngeal carcinoma (NPC). Previous studies have demonstrated that the developmental gene sex-determining region Y (SRY)-box 1 (SOX1) inhibits cervical and liver tumorigenesis by interfering with the Wnt/ $\beta$-catenin signaling pathway. However, the role of SOX1 in NPC remains unclear. This study investigates the function of SOX1 in NPC pathogenesis.

Results: Down-regulation of SOX1 was detected in NPC cell lines and tissues. Besides, quantitative methylation-specific polymerase chain reaction revealed that SOX1 promoter was hypermethylated in NPC cell lines. Ectopic expression of SOX1 in NPC cells suppressed colony formation, proliferation and migration in vitro and impaired tumor growth in nude mice. Restoration of SOX1 expression significantly reduced epithelial-mesenchymal transition, enhanced cell differentiation and induced cellular senescence. Conversely, transient knockdown of SOX 1 by siRNA in these cells partially restored cell proliferation and colony formation. Notably, SOX1 was found to physically interact with $\beta$-catenin and reduce its expression independent of proteasomal activity, leading to inhibition of Wnt/ 3 -catenin signaling and decreased expression of downstream target genes.
\end{abstract}

Conclusions: SOX1 decreases the expression of $\beta$-catenin in a proteasome-independent manner and reverses the malignant phenotype in NPC cells.

Keywords: SOX1, $\beta$-catenin, Methylation, Differentiation, NPC

\section{Background}

Nasopharyngeal carcinoma (NPC) is the most common head and neck cancer in southern China, Southeast Asia, the Arctic and the middle/northern regions of Africa. The incidence of NPC in southern China is approximately $25-50$ per 100,000 persons each year, which is 100-fold higher than that in Western countries [1-3]. The poor clinical outcome of NPC is largely attributable to resistance to therapies and metastasis [4]. Therefore, new strategies for safer and more effective treatment are urgently needed [5]. The molecular mechanisms underlying the pathogenesis of NPC are incompletely defined,

\footnotetext{
*Correspondence: liuq9@mail.sysu.edu.cn

${ }^{\dagger}$ Equal contributors

${ }^{2}$ Sun Yat-sen University Cancer Center, State Key Laboratory of Oncology in South China, Collaborative Innovation Center of Cancer Medicine,

Guangzhou 510060, China

${ }^{3}$ Institute of Cancer Stem Cell, Cancer Center, Dalian Medical University, Dalian 116044, China

Full list of author information is available at the end of the article
}

and an enhanced understanding of these will facilitate the development of novel therapeutics.

The family of sex-determining region Y (SRY)-box (SOX) proteins is a group of transcription factors that contain a highly conserved high-mobility group (HMG) DNAbinding domain. SOX family members play crucial roles in both embryonic and postnatal development and in stem cell regulation [6-8]. Moreover, several members of the SOX family have been implicated in cancer development [9-14]. For example, SOX10 facilitates the formation of a stable SOX10/T-cell factor (TCF)- $4 / \beta$-catenin complex, subsequently contributing to tumorigenesis in hepatocellular carcinoma (HCC). Similarly, SOX9 enhances tumor growth, angiogenesis and invasion in prostate cancer. However, SOX17 inhibits canonical Wnt/ $\beta$-catenin signaling and suppresses tumor growth in papillary thyroid carcinoma. Consistently, SOX1 is a tumor suppressor that is suppressed by 
hypermethylation of its promoter region in cervical and ovarian cancers. These findings are in accordance with the notion that hypermethylation of promoter regions of tumor suppressor genes is a major contributor to carcinogenesis $[15,16]$. For example, hypermethylation of the $\mathrm{p} 16^{I N K 4 a}$ promoter leads to decreased expression of its protein in NPC, further promoting tumorigenesis $[17,18]$. Additionally, aberrant promoter methylation of CDH13, DLEC1, CHFR and CDH1 has been implicated in tumorigenesis $[19,20]$. However, whether the methylation status of the SOX1 promoter is involved in the development of NPC remains to be elucidated.

The canonical Wnt signaling pathway is involved in various biological processes, including embryonic development, cell proliferation and stem cell maintenance [21]. Moreover, the dysregulation of Wnt signaling is implicated in human tumorigenesis. The central element of the canonical Wnt pathway is $\beta$-catenin, which forms complexes with TCF/lymphoid enhancer factor (LEF) HMG box transcription factors to stimulate the transcription of Wnt-responsive genes including CCND1, MYC and $C d x-1$ [22]. Previous studies have shown that SOX family members regulate $\mathrm{Wnt} / \beta$-catenin signaling through interaction with $\beta$-catenin. For example, SOX7 can suppress expression of Cyclin D1 and c-Myc via direct interaction with $\beta$-catenin, thereby inhibiting $\mathrm{Wnt} / \beta$-catenin signaling in endometrial, prostate and colon cancers $[23,24]$. Additionally, SOX1 competes with TCF/LEF by physically binding to $\beta$-catenin and therefore interfering with the activation of $\mathrm{Wnt} / \beta$-catenin signaling in $\mathrm{HCC}$ [25]. Therefore, we investigated whether SOX proteins regulate $\mathrm{Wnt} / \mathrm{\beta}$-catenin signaling in NPC.

In this study, we demonstrate that depletion of SOX1 is responsible for the malignant phenotype of NPC. We show that recovery of SOX1 expression leads to a downregulation of $\beta$-catenin that is independent of proteasomal activity. These new data show that SOX1 decreases $\beta$-catenin activity and reverses the malignant phenotype of NPC.

\section{Results}

Down-regulation of SOX1 in NPC cells and tissues is associated with promoter hypermethylation

We first examined the expression of SOX1 in six NPC cell lines and found that both mRNA and protein were barely detectable in all six cell lines. Conversely, both SOX1 mRNA and protein were highly expressed in NP69 cells, an immortalized human normal nasopharyngeal epithelial cell line (Figure 1A). We then confirmed these results in three primary NPC tissues and their corresponding adjacent non-tumor tissues using quantitative real-time polymerase chain reaction (qRT-PCR). SOX1 mRNA expression was significantly down-regulated in primary NPC tissues when compared with the adjacent non-tumor tissues (Figure 1B). We next asked whether the downregulation of SOX1 in NPC was caused by SOX1 promoter methylation. We determined the methylation status of the NPC cell lines by quantitative methylationspecific PCR (qMS-PCR). Hypermethylation was confirmed in the NPC cell lines that showed down-regulated SOX1 expression, whereas methylation was almost absent in NP69 cells (Figure 1C). To determine whether promoter methylation was involved in regulating SOX1, two NPC cell lines (CNE2 and HONE1) were treated with 5-AZA-2' -deoxycytidine (5-Aza-CdR), a DNA methyltransferase inhibitor. Re-expression of SOX1 was detected in both NPC cell lines when methylation was prevented (Figure 1D). These data suggest that the low levels of SOX1 expression were attributable to promoter methylation.

\section{Ectopic expression of SOX1 represses NPC cells proliferation and migration}

Since we observed a down-regulation of SOX1 in both NPC cell lines and tissues, we next determined whether overexpression of SOX1 could reverse the malignant phenotype of NPC cells. Virus-mediated overexpression of SOX1 in CNE2 and HONE1 cells was confirmed by western blot (WB) and immunofluorescence (IF) analysis (Figure 2A). Overexpression of SOX1 significantly decreased colony formation and proliferation in both CNE2 and HONE1 cells (Figure 2B and C). SOX1 overexpression also significantly decreased the percentage of Ki67 (+) cells in both CNE2 and HONE1 cells (Figure 2D). Furthermore, we found that the migration ability of both CNE2 and HONE1 cells was significantly suppressed when SOX1 was overexpressed (Figure 2E, and F and Additional file 1: Figure S1A).

\section{SOX1 impairs tumor formation in a xenograft model}

Next, we examined the influence of SOX1 on NPC cells tumor formation in vivo. CNE2 cells stably transfected with either empty vector or SOX1 were delivered subcutaneously into nude mice and tumor growth was monitored (Figure 3A). The tumors were harvested and weighed after 20 days of growth. When compared with the control group, the mean tumor volume (Figure 3B) and tumor weight (Figure $3 \mathrm{C}$ ) were significantly lower in mice inoculated with SOX1-overexpressing CNE2 cells. Tumor volume decreased from $1165.30 \pm 205.11 \mathrm{~mm}^{3}$ to $161.06 \pm$ $58.03 \mathrm{~mm}^{3}$ and weight decreased from $1.15 \pm 0.20 \mathrm{~g}$ to $0.15 \pm 0.04 \mathrm{~g}$ (both $p<0.001$ ). These results suggest that SOX1 impairs tumor growth in NPC cells in vivo.

\section{SOX1 overexpression reduces epithelial-mesenchymal transition, induces cell differentiation and enhances cellular senescence}

The functions of SOX1 in vitro were further investigated by overexpressing SOX1 in either CNE2 or HONE1 


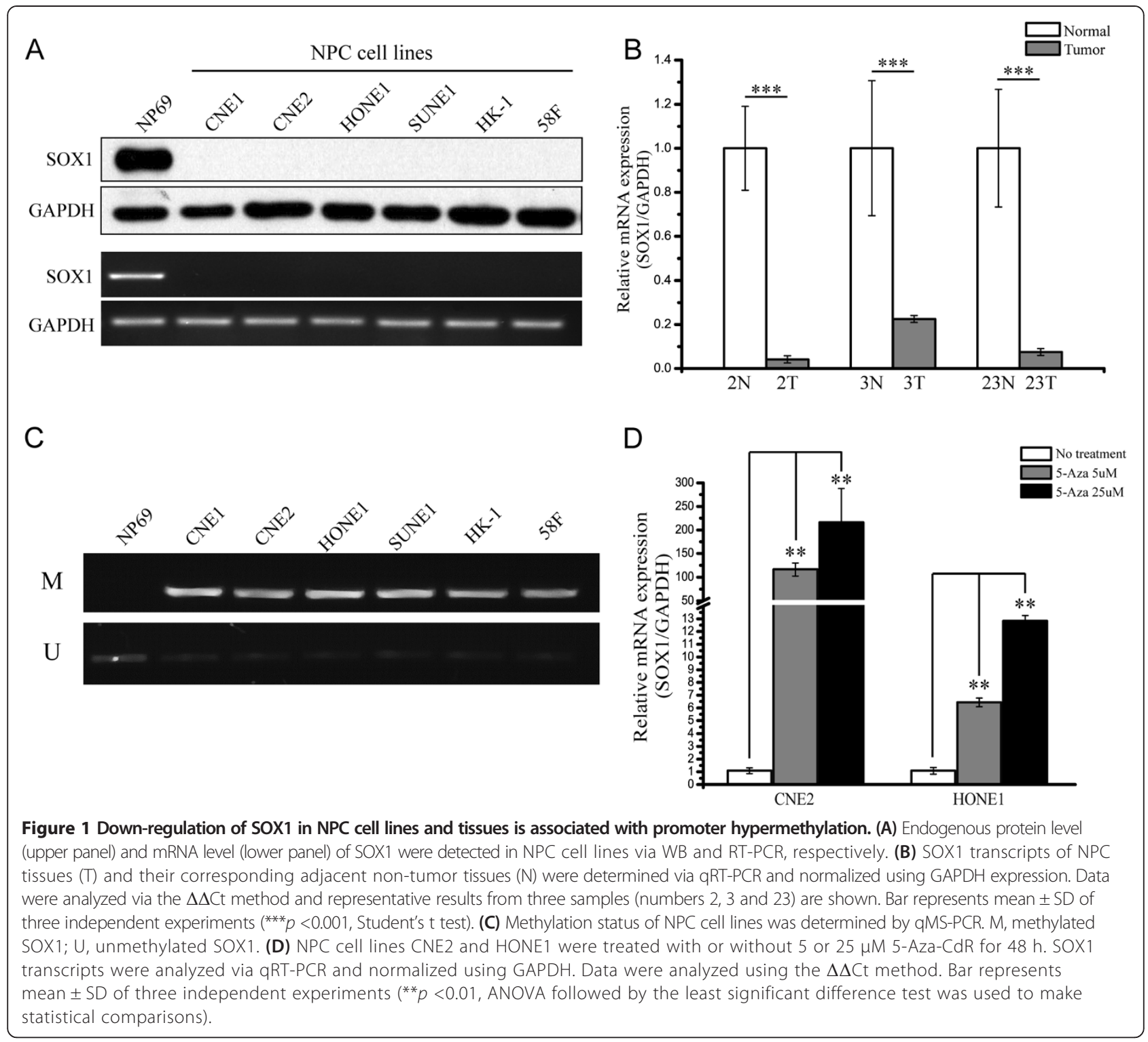

cells. As shown in Figure 4A and Additional file 2: Figure S2, overexpression of SOX1 down-regulated Vimentin and up-regulated E-cadherin in HONE1 and CNE2 cells, indicating that overexpression of SOX1 suppressed epithelial-mesenchymal transition (EMT). HONE1 is a poorly differentiated NPC cell line, and is histologically characterized by a round and cobblestone-like phenotype. Overexpression of SOX1 in HONE1 cells induced a morphology transition to a slender and fusiform appearance, which was defined as a differentiated phenotype (Figure 4B). This phenotype was similar to those of the well-differentiated NP69 and CNE1 cell lines [26]. Consistent with these morphological changes, expressions of cell differentiation markers such as Involucrin, CK8 and CK18 [27,28] were increased, whereas undifferentiation markers such as
CK19 and CK13 [29,30] were reduced (Figure 4B). Similar results could be observed in the CNE2 cells (Additional file 1: Figure S1B and S1C). Taken together, these data indicated that SOX1 overexpressing NPC cells were undergoing differentiation. As SOX1 has been implicated in the regulation of cell differentiation and EMT, we further examined the role of SOX1 in stem cell regulation in NPC. We found that sphere-forming ability was dramatically decreased by overexpression of SOX1 in both CNE2 (Additional file 3: Figure S3A, $p<0.01$ ) and HONE1 (Additional file 3: Figure $\mathrm{S} 3 \mathrm{~B}, p<0.01)$ cells. Meanwhile, colony-forming ability in soft agar was also decreased in these cells (Additional file 3: Figure S3C, both $p<0.05$ ). We also found that overexpression of SOX1 in HONE1 cells enhanced SA- $\beta$-gal staining (Figure 4C). 


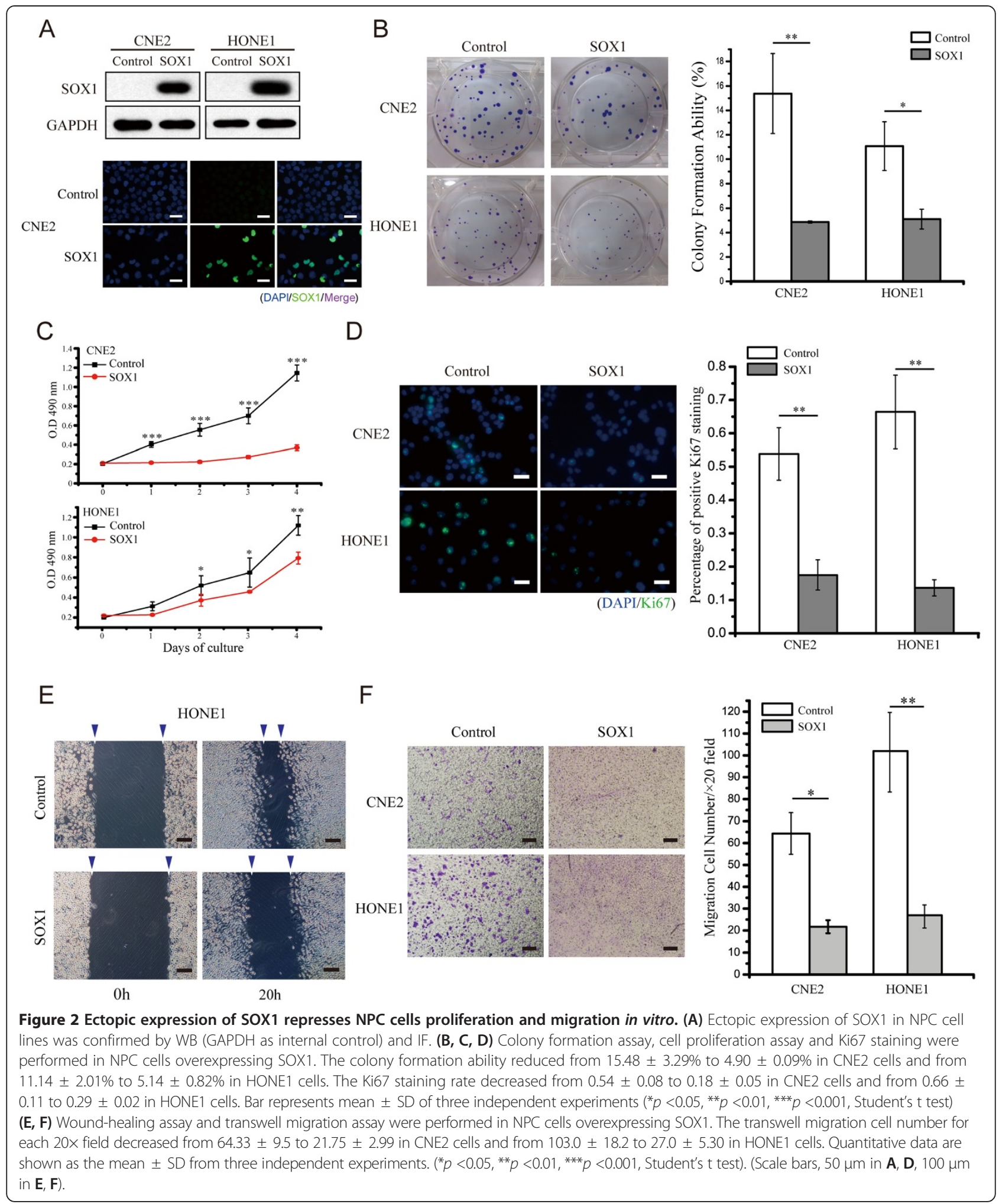

Knockdown of SOX1 partially recovers cellular proliferative capacity in SOX1 stably overexpressed NPC cells

To further demonstrate the anti-tumor function of SOX1, we knocked down SOX1 with transient siRNA transfection in CNE2 and HONE1 cells stably overexpressing SOX1. We confirmed SOX1 expression in both cell lines via WB (Figure 4D). Transient knockdown of SOX1 in these cells partially rescued cell proliferation, demonstrated by the 


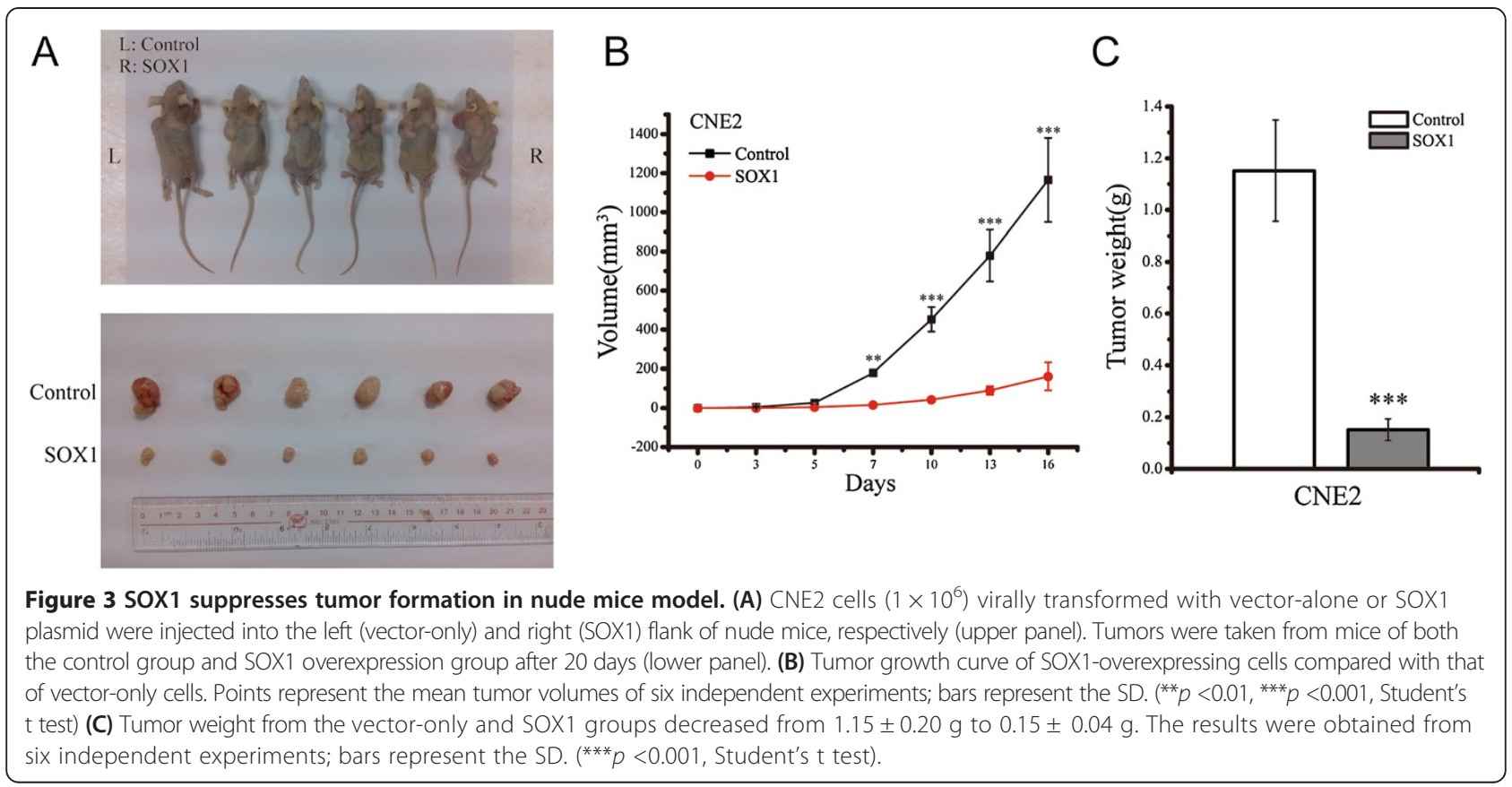

increased number of Ki67 (+) cells (Figure 4E) and the enhanced colony formation ability (Figure 4F).

\section{SOX1 interacts with $\beta$-catenin and induces a proteasome- independent down-regulation of $\beta$-catenin}

Previous studies have shown that SOX1 interacts with $\beta$-catenin in vitro to suppress $\beta$-catenin-mediated TCF/LEF signaling in HCC cell lines [25]. A co-immunoprecipitation assay identified $\beta$-catenin and SOX1 in an immunocomplex from HONE1 cell lysate, supporting the existence of this interaction in NPC cell lines (Figure 5A). We also examined the cellular localization of SOX1 and $\beta$-catenin using confocal microscopy, and identified SOX1 and $\beta$-catenin co-localization in HONE1 cells (Figure 5B). Interestingly, while expression of $\beta$-catenin was down-regulated in SOX1-overexpressing HONE1 cells (Figure 5C), transcription of the $\beta$-catenin gene was up-regulated (Figure $5 \mathrm{D}$ ), suggesting that SOX1 reduced expression of $\beta$-catenin at the protein level. Furthermore, the down-regulation of $\beta$-catenin induced by SOX1-overexpression was reversed by knockdown of SOX1 using siRNA (Figure 5E). Consistently, $\beta$-catenin was down-regulated in a dose-dependent manner with increasing amounts of SOX1 (Figure 5F). This phenomenon was not reversed by MG132, a specific proteasome inhibitor, suggesting that SOX1 downregulated $\beta$-catenin in a proteasome-independent manner (Figure 5F).

\section{SOX1 overexpression modulates the expression of cell cycle-regulating proteins}

To further explore the mechanisms by which SOX1 suppresses tumors, we measured the expression of downstream targets of Wnt/ $\beta$-catenin in SOX1-overexpressing HONE1 cells. A decrease in both c-Myc and Cyclin D1 protein was detected in SOX1-overexpressing HONE1 cells (Figure 6A). To further investigate the influence of SOX1 overexpression on the cell cycle, we evaluated cell cycle progression by flow cytometry. Ectopic expression of SOX1 increased the number of cells in the G1/G0 phase (from $67.77 \pm 0.9 \%$ to $71.82 \pm 1.05 \%$ ) and decreased the number of cells in the G2/M phase (from $12.7 \pm 0.10 \%$ to $9.3 \pm 0.10 \%$ ) in HONE1 cells (Figure 6B). To ascertain how SOX1 inhibited cell growth, protein levels of the cell cycle regulators p21, p27 and Cyclin E were examined using WB. In HONE1 cells, SOX1 overexpression significantly enhanced the expression of $\mathrm{p} 21$ and p27 but suppressed the expression of Cyclin E (Figure 6C).

\section{Discussion}

These new data provide compelling evidence to suggest that SOX1 suppresses tumorigenicity and regulates expression of $\beta$-catenin in NPC. Both NPC cell lines and NPC tissues showed decreased expression of SOX1 at the mRNA and protein levels. Our data further indicate that the decreased expression of SOX1 could be attributed to promoter hypermethylation. Moreover, overexpression of SOX1 in NPC cells reduced tumor formation and the tumor burden in vivo. We also found that SOX1 induced NPC cell differentiation and reduced EMT. Mechanistically, SOX1 inhibited the $\mathrm{Wnt} / \beta$-catenin signaling pathway by promoting $\beta$-catenin down-regulation in a proteasome-independent manner. Our study therefore reveals a novel mechanism by which SOX1 regulates 


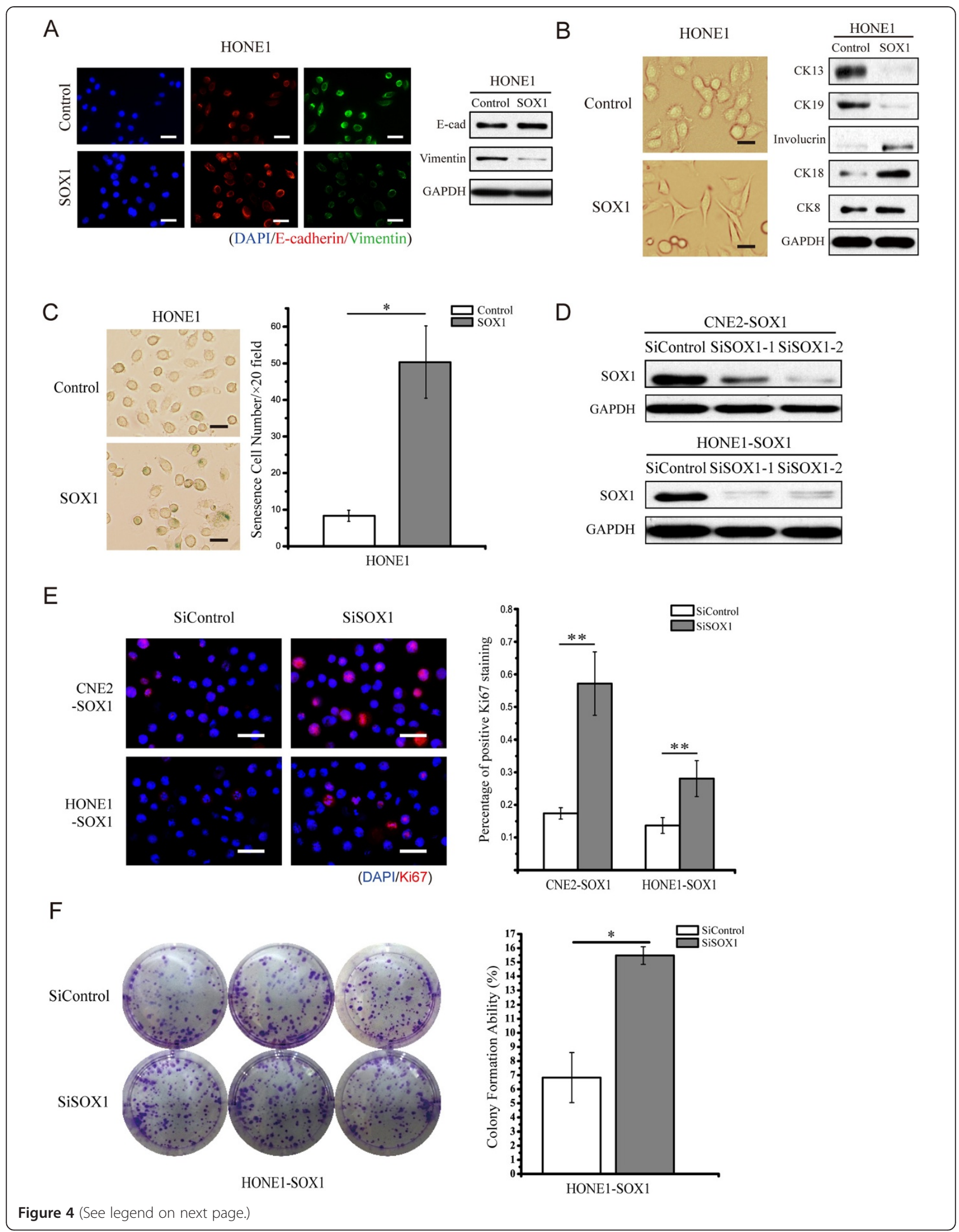


(See figure on previous page.)

Figure 4 SOX1 reduces EMT, stimulates cell differentiation and triggers cellular senescence, whereas transient knockdown of SOX1 partially reverses the malignant phenotype. (A) Presence of the EMT-related proteins E-cadherin and Vimentin was detected via IF and WB. IF (left panel) Blue, DAPI; Red, E-cadherin; Green, Vimentin. EMT-related markers were detected by WB (right panel) in HONE1 cells with forced SOX1 expression. GAPDH served as an internal control. (B) Morphology of differentiated HONE1 cells induced by SOX1 was observed under microscopy. WB was used to detect the cell surface markers related to cell differentiation in HONE1 cells with or without SOX1 overexpression. (C) HONE1 cells with or without SOX1 overexpression were fixed and stained for SA- $\beta$-gal activity. The number of senescent colonies within each 20x field of HONE1 cells increased from $8.33 \pm 1.53$ to $50.33 \pm 9.87$. Bar represents mean \pm SD of three independent experiments. $\left(^{*} p<0.05, S t u d e n t ' s t\right.$ test) (D) SOX 1 expression level in SOX1-overexpressing-NPC cells following transfection of SOX1-specific siRNA was determined using WB. (E, F) Ki67 staining rate and colony formation ability following knockdown of SOX 1 using siRNA in SOX1-overexpressing NPC cells. Ki67 staining rate increased from $0.17 \pm 0.02$ to $0.49 \pm 0.10$ in CNE2 cells and from $0.14 \pm 0.02$ to $0.28 \pm 0.06$ in HONE1 cells. Colony formation ability increased from $6.82 \pm 1.77 \%$ to $15.47 \pm 5.28 \%$ in HONE1 cells. Bar represents mean \pm SD of three independent experiments $\left(^{*} p<0.05,{ }^{* *} p<0.01\right.$, Student's t test). (Scale bars, $50 \mu m$ in $\mathbf{A}, 25 \mu m$ in $\mathbf{B}, \mathbf{C}, 50 \mu \mathrm{m}$ in $\mathbf{E})$.

tumorigenesis by interfering with $\mathrm{Wnt} / \beta$-catenin signaling in NPC.

A previous study has shown that another member of the SOX family, SOX11, can also act as a tumor suppressor in NPC cells. Down-regulation of SOX11 mRNA expression was observed in NPC tissues that displayed methylation of SOX11 promoters. CNE2 cells treated with the hypomethylating agent 5 -Aza-CdR recovered expression of SOX11 and experienced inhibited cell growth and invasion [31]. Consistently, we found that SOX1 was also down-regulated via promoter hypermethylation in NPC (Figure 1C and D). Overexpression of SOX1 subsequently decreased expression of $\beta$-catenin and suppressed the Wnt/ $\beta$-catenin signaling pathway, thereby reversing the malignant phenotype of NPC cells. Our findings suggest that an inhibitor of promoter hypermethylation that specifically targets tumor suppressor genes could be beneficial for future NPC treatment strategies.

Functional analysis of the SOX family as transcription factors has demonstrated their important roles in stem cell regulation [8]. These discoveries have linked SOX family proteins with $\mathrm{Wnt} / \beta$-catenin-mediated regulation of stem cell self-renewal and differentiation [21]. For instance, SOX3 and SOX17 were first reported to regulate the canonical Wnt/ $\beta$-catenin signaling pathway in Xenopus embryos [32]. More recently, the link between SOX family proteins and $\mathrm{Wnt} / \beta$-catenin signaling has been implicated in tumorigenesis [14,23]. For example, SOX7 and SOX17 have been suggested to suppress tumor growth through an interaction with $\beta$-catenin in various cancer types. Furthermore, overexpression of SOX1 attenuated $\beta$-catenin-mediated TCF/LEF signaling via binding with $\beta$-catenin and competition for TCF/LEF binding sites in HCC [25].

Previous reports showed that SOX1 acted as a tumor suppressor by interacting with $\beta$-catenin in cervical and liver cancers $[25,33]$. SOX1 interfered with Wnt/ $\beta$-catenin signaling by competing for TCF/LEF binding sites in HCC. Consistently, our results demonstrate that SOX1 can suppress malignant properties and induce decreased expression of $\beta$-catenin in NPC (Figures 2, 3, 4 and $5 \mathrm{C}$ ). In the canonical Wnt/ $\beta$-catenin signaling pathway, $\beta$-catenin degradation occurs predominantly through a multiprotein destruction complex comprising factors including tumor suppressors adenomatous polyposis coli (APC), Axin, the kinases casein kinase 1 (CK1) and glycogen synthase kinase $3 \beta$ (GSK-3 $\beta)$. APC recruits $\beta$-catenin to the destruction complex, where it is phosphorylated at $\mathrm{N}$-terminal serine and threonine residues by CK1 and GSK-3 $\beta$, leading to its ubiquitination and subsequent proteasomal degradation [34]. In the present study, we found that levels of $\beta$-catenin protein, but not mRNA, were down-regulated by SOX1 overexpression in a dose-dependent manner (Figure 5F). Furthermore, the down-regulation of $\beta$-catenin induced by SOX1 could not be reversed by MG132, suggesting that SOX1 promoted the down-regulation of $\beta$-catenin through a proteasome-independent pathway. We also found that SOX1 and $\beta$-catenin were present in the same immunocomplex and also co-localized in HONE1 cells (Figure 5A and B). The co-localization of SOX1 and $\beta$-catenin partially overlaps with the nuclear DNA signal, suggesting that the co-localization may reside in the cytoplasm and that SOX1 can induce down-regulation of $\beta$-catenin in the cytoplasm. These results are consistent with previous reports that $\beta$-catenin is degraded in the cytoplasm. Here, we have shown for the first time that SOX proteins can attenuate $\mathrm{Wnt} / \beta$-catenin signaling independent of the proteasome pathway, and will further investigate the mechanism(s) underlying this SOX1-mediated $\beta$-catenin down-regulation in future studies.

Dysregulation of the cell cycle contributes to the etiology of several diseases, including cancer. It has been well established that the $\mathrm{Wnt} / \beta$-catenin signaling pathway is involved in cell cycle regulation in various cancers $[21,35]$. Moreover, as downstream targets of the Wnt/ $\beta$-catenin signaling pathway, Cyclin D1 and c-Myc play important roles in cell cycle regulation. We found that overexpression of SOX1 decreased $\beta$-catenin in NPC cells, and that down-regulation of Cyclin D1 and c-Myc as well as up-regulation of p21 and p27 also occurred in SOX1overexpressing HONE1 cells (Figure 6A and $\mathrm{C}$ ). 


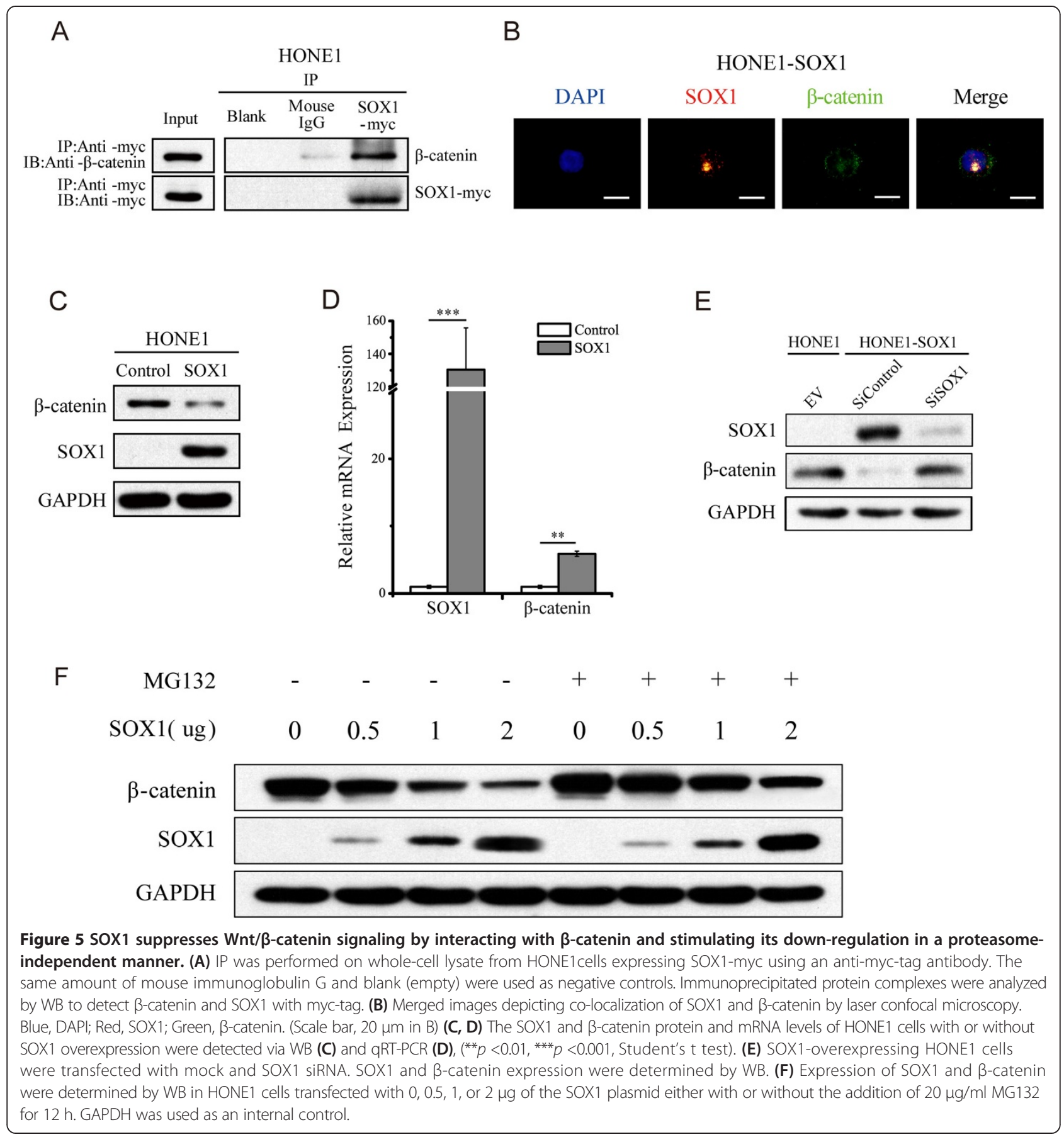

Meanwhile, ectopic expression of SOX1 in HONE1 cells increased the number of cells in the G1/G0 phase while decreasing the number of cells in the G2/M phase (Figure 6B). These results suggested that SOX1 inhibited cell cycle progression by attenuating Wnt/ $\beta$-catenin signaling. Additional evidence of this is supplied by studies of neural system development, where SOX1 binding to $\beta$ catenin suppressed $\beta$-catenin-mediated TCF/LEF signaling, thereby promoting cell cycle exit [36]. Further studies are needed to investigate the mechanism involved in the cell cycle regulation by SOX1.

Previous reports have demonstrated that SOX1 regulates the differentiation of neural progenitor cells by directly binding to $\beta$-catenin and suppressing $\beta$-cateninmediated TCF/LEF signaling [36,37]. These reports are consistent with our finding that overexpression of SOX1 triggers differentiation of CNE2 and HONE1 cells, which are both poorly differentiated NPC cell lines (Figure 4B 

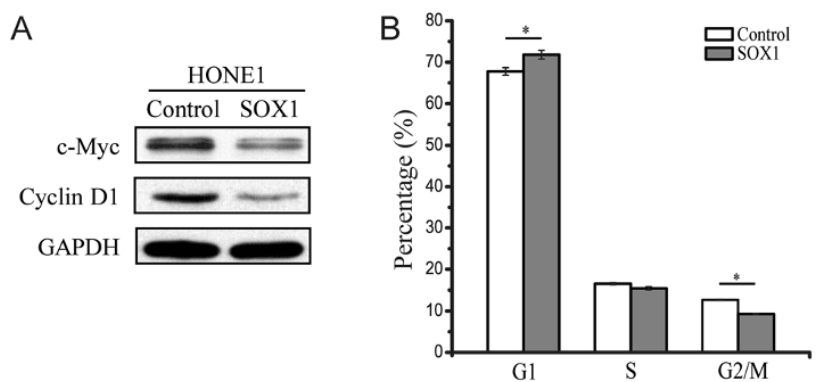

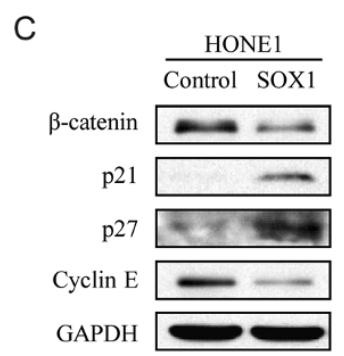

Figure 6 SOX1 inhibits Wnt/B-catenin downstream genes and modulates the expression of molecules involved in the cell cycle. (A) WB for expression of c-Myc and Cyclin D1 in SOX1-overexpressing HONE1 cells. (B) Flow cytometry cell cycle analysis of HONE1 cells overexpressing SOX1. The percentage of cells in $\mathrm{G} 1$ phase increased from $67.77 \pm 0.9 \%$ to $71.82 \pm 1.05 \%$ while the percentage of cells in G2/M phase decreased from $12.7 \pm 0.10 \%$ to $9.3 \pm 0.10 \%$ in HONE1 cells overexpressing SOX1. Bar represents mean \pm SD of three independent experiments. ${ }^{*} p<0.05$, Student's $t$ test). (C) Proteins involved in the cell cycle after SOX1 expression was forced in HONE1 cells were identified via WB. GAPDH was used as an internal control.

and Additional file 1: Figure S1B and S1C). Further investigation is needed to explore how SOX1 regulates cancer cell differentiation and whether the interaction between SOX1 and $\beta$-catenin is involved in this regulation. Our findings will provide new guidance for NPC treatment and improve prognoses in the future.

\section{Conclusion}

SOX1 expression is frequently down-regulated in NPC because of promoter hypermethylation. Overexpression of SOX1 reverses the malignant phenotype of NPC cells by suppressing cell migration and EMT and inducing cell senescence and cell differentiation. The potential mechanism by which SOX1 suppresses Wnt/ $\beta$-catenin signaling is by down-regulation of $\beta$-catenin and subsequent inhibition of downstream gene expression. These findings indicate that SOX1 plays a pivotal role as a tumor suppressor in NPC development.

\section{Methods}

\section{Cell lines and cell culture}

Six NPC cell lines (CNE1, CNE2, HONE1, SUNE1, 58 F and HK1) and an immortalized normal nasopharyngeal epithelial cell line (NP69) were used in this study. All cells were obtained from the State Key Laboratory of Oncology in South China. CNE1 and CNE2 were cultured in RPMI-1640 (Gibco, Life Technologies, CA, USA) containing $10 \%$ fetal calf serum (Gibco). HONE1, SUNE1, $58 \mathrm{~F}$ and HK1 were cultured in RPMI-1640 containing 10\% fetal bovine serum (Hyclone, South America). NP69 cells were propagated in defined keratinocyte-serum-free medium (K-SFM; Gibco, Life Technologies, Basel, Switzerland) supplemented with growth factors. Virus-converted cells with and without stable SOX1 overexpression (CNE2pLVPT and CNE2-SOX1, HONE1-pLVPT and HONE1SOX1) were maintained in incomplete medium with
$1.5 \mu \mathrm{g} / \mathrm{ml}$ puromycin. All cells were maintained at $37^{\circ} \mathrm{C}$ in a humidified atmosphere containing $5 \% \mathrm{CO}_{2}$.

\section{Bisulfite conversion and quantitative methylation-specific} polymerase chain reaction (qMS-PCR)

qMS-PCR was performed using the EZ DNA MethylationGold $^{\text {mix }}$ Kit (ZYMO Research, CA, USA) according to the manufacturer's protocol. The primer sequence for qMSPCR has been described [38]. Methylated primers for each construct were as follows: hSOX1, forward $5^{\prime}$-CGTTT TTTTTTTTTCGTTATTGGC-3', reverse 5'-CCTACGC TCGATCCTCAACG-3'; unmethylated primers for hSOX1, forward $5^{\prime}$-TGTTTTTTTTTTTTTGTTATTGGTG-3', reverse 5'-CCTACACTCAATCCTCAACAAC-3'. All qMSPCR data were obtained from at least three independent experiments to ensure reproducibility.

\section{RNA isolation, reverse-transcription PCR (RT-PCR) and real-time quantitative RT-PCR}

RNA was isolated from each tissue or cell sample using Trizol reagent (Invitrogen, CA, USA) according to the manufacturer's protocol. A 2- $\mu$ g aliquot of total RNA from each sample was used for cDNA synthesis with SuperScript ${ }^{\circ}$ III RT (Invitrogen) and an oligo-dT primer. PCR was performed at $95^{\circ} \mathrm{C}$ for $5 \mathrm{~min}$, then 30 cycles $\left(95^{\circ} \mathrm{C}\right.$ for $5 \mathrm{sec}, 55-68^{\circ} \mathrm{C}$ for $15 \mathrm{sec}$ and $72^{\circ} \mathrm{C}$ for $\left.1 \mathrm{~min}\right)$ with a final extension of $10 \mathrm{~min}$ at $72^{\circ} \mathrm{C}$. The primer sequence for SOX1 was as follows: forward 5 -AAGGT GAAGGTCGGAGTCAAC-3', reverse 5'-GGGGTCAT TGATGGCAACAATA-3'. Real-time PCR was performed using Platinum ${ }^{\circ}$ SYBR $^{\circ}$ Green qPCR SuperMix (Invitrogen) and analyzed on a CFX96 Real-Time System (Bio-Rad, CA, USA). Glyceraldehyde-3-phosphate dehydrogenase gene (GAPDH) was used as an internal control. The difference of real-time PCR cycle number (Ct value) between the target gene and GAPDH was quantified using the $\Delta \Delta C T$ method. 


\section{Western blot (WB) and antibodies}

The WB procedure was performed as described previously [39] with some modifications. Briefly, after transient transfection with plasmids, cells were harvested and lysed in a lysis buffer containing a cocktail of protease inhibitors. After centrifugation at 12,000 rpm for $15 \mathrm{~min}$ at $4{ }^{\circ} \mathrm{C}$, supernatants were collected, mixed with dithiothreitol, and used for WB. The ProteoExtract ${ }^{\circ}$ Subcellular Proteome Extraction Kit (Millipore) was used for extraction of subcellular fractions following the manufacturer's protocol. Equal amounts of protein extract were electrophoresed in 10\% SDS-PAGE gels and then transferred to nitrocellulose membranes. The membranes were blocked with $5 \%$ bovine serum albumin (BSA) at room temperature (RT) for $1 \mathrm{~h}$, incubated with the primary antibody overnight at $4{ }^{\circ} \mathrm{C}$, and incubated with the secondary antibody at room temperature for $1 \mathrm{~h}$. Blots were washed in Tris-buffered saline with $0.1 \%$ Tween-20 and proteins were visualized by chemiluminescence. The following antibodies were used for WB: anti-SOX1 (IB 1:2000, Epitomics, CA, USA), anti-CK19 (1:8000, Epitomics), anti-CK18 (1:4000, Epitomics), anti-CK13 (1:4000, Epitomics), anti-CK8 (1:4000, Epitomics), anti-Involucrin (1:4000, Abcam, MA, USA), anti-GAPDH (1:10000, ProteinTec Group, IL, USA), anti-c-Myc (N-262) (1:2000, Santa Cruz Biotechnology, CA, USA), and anti- $\beta$-catenin (1:2000, Upstate, NY, USA).

\section{Immunofluorescence (IF)}

The IF procedure was performed as described previously [40] with some modifications. Briefly, cells were seeded on slides at $50 \%$ confluency in 6-well plates for $24 \mathrm{~h}$ before fixation. All the following procedures were performed at room temperature. Cells on slides were fixed with $2 \%$ formalin for $15 \mathrm{~min}$. After rinsing with phosphatebuffered saline (PBS), slides were permeabilized in $0.25 \%$ Triton-X-100 buffer for $15 \mathrm{~min}$. After rinsing with PBS, non-specific sites on the slides were blocked with $3 \%$ BSA for 20 min. Slides were then incubated with primary antibodies diluted in 3\% BSA for $1 \mathrm{~h}$ : anti-SOX1 (1:200), $\beta$-catenin (1:200), Ki67 (1:200, Santa Cruz Biotechnology). After rinsing with PBS, the slides were incubated with secondary antibody (1:200) for $1 \mathrm{~h}$ in the dark. The slides were rinsed with PBS, stained with $100 \mathrm{ng} / \mathrm{ml}$ 4',6-diamidino-2-phenylindole for $5 \mathrm{~min}$, and rinsed with PBS. The slides were dried and placed on embedding medium on cover slides, and then observed under a fluorescence microscope.

\section{Immunoprecipitation}

Immunoprecipitation was conducted according to a previous report [26]. Polyclonal Anti-c-myc-tag (Sigma-Aldrich, MO, USA) antibody was cross-linked to protein $G$ beads (Invitrogen) according to the manufacturer's protocol. Cell lysate from NPC cells transfected with SOX1-myc plasmid was pre-cleared with IgG beads-protein $\mathrm{G}$ for $2 \mathrm{~h}$ and then incubated with c-myc-tag-beads overnight at $4^{\circ} \mathrm{C}$. C-myctag-immunoprecipitated complexes were washed five times with immunoprecipitation buffer $(10 \mathrm{mM}$ Tris/ $/ \mathrm{HCl}$, pH 7.8; 1 mM EDTA; 150 mM NaCl; $1 \mathrm{mM} \mathrm{NaF;} \mathrm{0.5 \%}$ Nonidet P-40; $0.5 \%$ glucopyranoside; $1 \mu \mathrm{g} / \mathrm{ml}$ aprotinin; and $0.5 \mathrm{mM}$ phenylmethylsulfonyl fluoride). Proteins were eluted from beads by boiling in loading buffer, and then subjected to WB. Anti- $\beta$-catenin and anti-c-myc-tag antibodies were used to detect $\beta$-catenin and SOX1-myc.

\section{Colony formation assay}

The colony formation assay was performed according to the procedure reported previously [41]. Briefly, cells were trypsinized, resuspended as single cells and plated onto 6-well plates with 500 cells per well. After 10 days, the colonies were fixed with $2 \%$ methanol and stained with crystal violet. Colonies with more than 50 cells were counted under the microscope. The cloning efficiency was calculated using the whole area of the colonies.

\section{Wound-healing assay and transwell migration assay}

For the wound-healing assay, CNE2-EV\&SOX1 and HONE1-EV\&SOX1 cells were seeded in a 6-well plate at $90 \%$ confluence. The day before the assay, cells were starved with serum-free medium overnight. A sterile 200- $\mu$ l pipet tip was used to scratch three separate wounds through the cells moving perpendicular to the line drawn with a marker on the bottom of the dish. Then, cells were gently rinsed with PBS and $1.5 \mathrm{ml}$ of media was added. Pictures were taken using a phase contrast microscope at $0,12,16$ and $20 \mathrm{~h}$. For the transwell migration assay, $800 \mu \mathrm{l}$ of normal medium was added to the lower chamber in a 24-well plate, and $200 \mu \mathrm{l}$ cell suspension $\left(1 \times 10^{5}\right.$ cells $)$ in SFM was added to the upper chamber. After incubation for $24 \mathrm{~h}\left(37^{\circ} \mathrm{C}, 5 \% \mathrm{CO}_{2}\right)$, the membrane was fixed with $2 \%$ methanol and stained with crystal violet. Pictures were taken under a microscope and the stained cells in the lower side of the membrane were counted.

\section{In vivo tumorigenicity}

CNE2 cells $\left(5 \times 10^{5}\right)$ transfected with empty vector or SOX1-expressing plasmid were injected subcutaneously into the left and right flanks of 6-week-old nude mice (Guangdong Medical Laboratory Animal Center, Guangdong, China). The tumor volume was calculated as $0.5 \times \mathrm{L} 1 \times(\mathrm{L} 2)^{2}$, where L1 is the long axis and L2 is the short axis of the tumor. The developing tumors were observed every third day. After 20 days, the mice were sacrificed at the end of follow-up. The animal study protocol was approved by the Institutional Animal Care and Use Committee. 


\section{Senescence-associated $\beta$-galactosidase staining} CNE2-EV\&SOX1 and HONE1-EV\&SOX1 cells were plated in a 6-well plate. The Senescence- $\beta$-Gal Staining Kit (Beyotime Institute of Biotechnology, Jiangsu, China) was used according to the manufacturer's instructions. Briefly, cells were fixed with fixation buffer for $10 \mathrm{~min}$ after rinsing three times in PBS for $5 \mathrm{~min}$ at RT. The cells were stained with staining buffer at $37^{\circ} \mathrm{C}$ overnight covered with plastic wrap. The cells were rinsed three times in PBS for $5 \mathrm{~min}$ at RT and observed under a microscope.

\section{Flow cytometry for cell cycle analyses}

Cells were harvested by digestion, washed with pre-chilled $\mathrm{PBS}$, fixed with $70 \%$ ethanol at $4^{\circ} \mathrm{C}$ for $30 \mathrm{~min}$, then stained with propidium iodide (Sigma-Aldrich, $50 \mathrm{mg} / \mathrm{ml}$ ) for $30 \mathrm{~min}$ at $4^{\circ} \mathrm{C}$ in the dark. Stained cells were subjected to flow cytometry analysis to measure the sub-G1 fraction.

\section{Sphere formation assay}

Sphere formation assays were performed as per a previous report [42]. Cells were seeded into an ultralow attachment 24-well plate (Corning) at 500 cells per well. Cells were grown in a DMEM/F12 medium (Gibco), supplemented with B27 (Invitrogen), EGF $(20 \mathrm{ng} / \mathrm{ml})$, bFGF $(20 \mathrm{ng} / \mathrm{ml}, \mathrm{BD}$ Biosciences), and heparin $(0.5 \mathrm{U} / \mathrm{ml}$, Sigma). Fresh medium was added $(200 \mu \mathrm{l} /$ well $)$ and the plates were gently shaken every other day. Spheres were counted following 10-15 days of culture.

\section{Soft agar colony formation assay}

Soft agar colony formation assays were conducted according to a previous report [43]. Briefly, cells were resuspended in medium containing $0.6 \%$ agarose ( 500 cells/well) and seeded into a 6-well plate coated with $1.2 \%$ agarose. Then $2 \mathrm{ml}$ culture medium was added on the top to maintain moisture and nutrient availability. Colonies were counted after culture for 2-3 weeks.

\section{Statistical analysis}

Statistical analyses were performed using the SPSS software, version 16.0 (SPSS Inc.) or with GraphPad Prism 5.0 (GraphPad Software, Inc.). The unpaired Student's t test was used to perform a statistical comparison between two groups. The ANOVA test was used when performing multiple comparisons. The level of significance was set at $p<0.05$. Error bars represent the standard deviation of the mean (SD), and each experiment was completed at least twice with samples in triplicate.

\section{Additional files}

Additional file 1: Figure S1. Ectopic expression of SOX1 represses CNE2 cells migration and induces cell differentiation in vitro. (A) The wound-healing assay performed in CNE2 cells overexpressing SOX1. (B) Morphology of differentiated CNE2 cells induced by SOX1 was observed under microscopy. (C) WB was used to detect the cell surface markers related to cell differentiation in CNE2 cells with or without SOX1 overexpression. (Scale bars, $200 \mu \mathrm{m}$ in $\mathrm{A}$ and $25 \mu \mathrm{m}$ in B).

Additional file 2: Figure S2. SOX1 reduces epithelial-mesenchymal transition in CNE2 cells. (A) Presence of the EMT-related proteins E-cadherin and Vimentin were detected via IF in CNE2 cells. Blue, DAPI; Red, E-cadherin; Green, Vimentin. (B) EMT-related markers were detected by WB in CNE2 cells with forced SOX1 expression. GAPDH served as an internal control.

Additional file 3: Figure S3. Overexpression of SOX1 negative regulated the stem cell ability of NPC cells. (A, B) The sphere formation ability reduced significantly in both CNE2 (from $64.5 \pm 9.95$ to $32 \pm 10.23$ per 500 cells, ${ }^{* *} p<0.01$, Student's t test) and HONE1 (from $31.25 \pm 5.12$ to $12.75 \pm 3.50$ per 500 cells, ${ }^{* *} p<0.01$ ). (C) The colony formation ability in soft agar dramatically decreased upon SOX1 overexpression, from $19.3 \pm$ 4.5 to $5.3 \pm 1.5$ each $20 \times$ field in CNE2 and from $20.7 \pm 5.0$ to $7.7 \pm 2.1$ each $20 \times$ field in HONE1, both ${ }^{*} p<0.05$, Student's t test. Quantitative data were shown as the mean \pm SD from three independent experiments. (Scale bars, $100 \mu \mathrm{m}$ in $\mathrm{A}, \mathrm{B}, 1000 \mu \mathrm{m}$ in $\mathrm{C})$.

\section{Abbreviations}

BSA: Bovine serum albumin; CSC: Cancer stem cell; EMT: Epithelial-mesenchymal transition; HCC: Hepatocellular carcinoma; HMG: High-mobility group; IP assay: Immunoprecipitation assay; LEF: Lymphoid enhancer factor; NPC: Nasopharyngeal carcinoma; PBS: Phosphate-buffered saline; PCR: Polymerase chain reaction; GMS-PCR: Quantitative methylation-specific PCR; RT-PCR: Quantitative real-time PCR; SPARC: Secreted protein, acidic, cysteine-rich; TCF: T-cell factor.

\section{Competing interests}

We declare that we have no financial or personal relationships with other people or organizations that could inappropriately influence our work. There is no professional or other personal interest of any nature or kind in any product, service, and/or company that could be construed as influencing the position presented in, or the review of, the manuscript entitled.

\section{Authors' contributions}

The work presented here was carried out in collaboration between all authors. QL, ZG and JLZ defined the research theme. JW and MY designed methods and experiments. JLZ carried out the laboratory experiments, analyzed the data, interpreted the results and wrote the paper. FMZ, JRP, YDX and YH co-worked on associated experimental data collection and their interpretation. JLZ, JW and HFW co-designed experiments, and discussed analyses, interpretation and presentation. All authors have approved the manuscript submission.

\section{Acknowledgments}

This research work was supported by the National Nature Science Foundation of China (No. 30873009 to Z.G.), National Basic Research Program of China (973 Program, No. 2012CB967000 to Q. Liu), National Natural Science Foundation of China (No. 81130040 to Q. Liu), Program for Chang Jiang Scholars and Innovative Research Team in University (No. IRTI 3049 to Q. Liu), and the Liaoning (NSF2014029102 to Q. Liu). We would like to thank the colleagues in Liu Q's lab in Sun Yat-sen University Cancer Center for their training, guidance, support and advice. We also thank Fei-meng Zheng, Cai-feng Yue, Wei Zhang, Min Yan and Yan Zhang for their help as we performed the research.

\section{Author details}

'Department of Otorhinolaryngology, Sun Yat-sen Memorial Hospital, Sun Yat-sen University, Guangzhou 510120, China. ${ }^{2}$ Sun Yat-sen University Cancer Center, State Key Laboratory of Oncology in South China, Collaborative Innovation Center of Cancer Medicine, Guangzhou 510060, China. ${ }^{3}$ Institute of Cancer Stem Cell, Cancer Center, Dalian Medical University, Dalian 116044 , China.

Received: 14 February 2014 Accepted: 28 October 2014

Published: 26 November 2014 


\section{References}

1. Tao Q, Chan AT: Nasopharyngeal carcinoma: molecular pathogenesis and therapeutic developments. Expert Rev Mol Med 2007, 9:1-24.

2. Lee AW, Fee WE Jr, Ng WT, Chan LK: Nasopharyngeal carcinoma: salvage of local recurrence. Oral Oncol 2012, 48:768-774.

3. Wei KR, Zheng RS, Zhang SW, Liang ZH, Ou ZX, Chen WQ: Nasopharyngeal carcinoma incidence and mortality in china in 2010. Chin J Cancer 2014, 33:381-387.

4. Yoshizaki T, Ito M, Murono S, Wakisaka N, Kondo S, Endo K. Current understanding and management of nasopharyngeal carcinoma. Auris Nasus Larynx 2012, 39:137-144.

5. Cao SM, Simons MJ, Qian CN: The prevalence and prevention of nasopharyngeal carcinoma in china. Chin J Cancer 2011, 30:114-119.

6. Gubbay J, Collignon J, Koopman P, Capel B, Economou A, Munsterberg A, Vivian N, Goodfellow P, Lovell-Badge R: A gene mapping to the sex-determining region of the mouse $Y$ chromosome is a member of a novel family of embryonically expressed genes. Nature 1990, 346:245-250.

7. Chew LJ, Gallo V: The Yin and Yang of Sox proteins: Activation and repression in development and disease. J Neurosci Res 2009, 87:3277-3287.

8. Sarkar A, Hochedlinger $K$ : The sox family of transcription factors: versatile regulators of stem and progenitor cell fate. Cell Stem Cell 2013, 12:15-30

9. Zhou D, Bai F, Zhang X, Hu M, Zhao G, Zhao Z, Liu R: SOX10 is a novel oncogene in hepatocellular carcinoma through Wnt/beta-catenin/TCF4 cascade. Tumour Biol 2014, 35:9935-9940.

10. Wang H, Leav I, Ibaragi S, Wegner M, Hu GF, Lu ML, Balk SP, Yuan X: SOX9 is expressed in human fetal prostate epithelium and enhances prostate cancer invasion. Cancer Res 2008, 68:1625-1630.

11. Rudin CM, Durinck S, Stawiski EW, Poirier JT, Modrusan Z, Shames DS, Bergbower EA, Guan Y, Shin J, Guillory J, Rivers CS, Foo CK, Bhatt D, Stinson J, Gnad F, Haverty PM, Gentleman R, Chaudhuri S, Janakiraman V, Jaiswal BS, Parikh C, Yuan W, Zhang Z, Koeppen H, Wu TD, Stern HM, Yauch RL, Huffman KE, Paskulin DD, Illei PB, et al: Comprehensive genomic analysis identifies SOX2 as a frequently amplified gene in small-cell lung cancer. Nat Genet 2012,44:1111-1116.

12. Mathews LA, Hurt EM, Zhang X, Farrar WL: Epigenetic regulation of CpG promoter methylation in invasive prostate cancer cells. Mol Cancer 2010, 9:267.

13. Yin D, Jia Y, Yu Y, Brock MV, Herman JG, Han C, Su X, Liu Y, Guo M: SOX17 methylation inhibits its antagonism of Wnt signaling pathway in lung cancer. Discov Med 2012, 14:33-40.

14. Li JY, Han C, Zheng LL, Guo MZ: Epigenetic regulation of Wnt signaling pathway gene SRY-related HMG-box 17 in papillary thyroid carcinoma. Chin Med J (Engl) 2012, 125:3526-3531.

15. Dawson MA, Kouzarides T: Cancer epigenetics: from mechanism to therapy. Cell 2012, 150:12-27.

16. Herman JG, Baylin SB: Gene silencing in cancer in association with promoter hypermethylation. N Engl J Med 2003, 349:2042-2054.

17. Zhong J, Min L, Huang H, Li L, Li D, Li J, Ma Z, Dai L: EZH2 regulates the expression of p16 in the nasopharyngeal cancer cells. Technol Cancer Res Treat 2013, 12:269-274

18. Tian F, Yip SP, Kwong DL, Lin Z, Yang Z, Wu WW: Promoter hypermethylation of tumor suppressor genes in serum as potential biomarker for the diagnosis of nasopharyngeal carcinoma. Cancer Epidemio/ 2013, 37:708-713.

19. Ayadi W, Karray-Hakim H, Khabir A, Feki L, Charfi S, Boudawara T, Ghorbel A, Daoud J, Frikha M, Busson P, Hammami A: Aberrant methylation of p16, DLEC1, BLU and E-cadherin gene promoters in nasopharyngeal carcinoma biopsies from Tunisian patients. Anticancer Res 2008, 28:2161-2167.

20. Sun D, Zhang Z, Van do N, Huang G, Ernberg I, Hu L: Aberrant methylation of $\mathrm{CDH} 13$ gene in nasopharyngeal carcinoma could serve as a potential diagnostic biomarker. Oral Oncol 2007, 43:82-87.

21. Clevers $\mathrm{H}$ : Wnt/beta-catenin signaling in development and disease. Cell 2006, 127:469-480.

22. Lickert H, Domon C, Huls G, Wehrle C, Duluc I, Clevers H, Meyer BI, Freund JN, Kemler R: Wnt/(beta)-catenin signaling regulates the expression of the homeobox gene Cdx1 in embryonic intestine. Development 2000, 127:3805-3813.

23. Chan DW, Mak CS, Leung TH, Chan KK, Ngan HY: Down-regulation of Sox7 is associated with aberrant activation of Wnt/b-catenin signaling in endometrial cancer. Oncotarget 2012, 3:1546-1556.

24. Guo L, Zhong D, Lau S, Liu X, Dong XY, Sun X, Yang WW, Vertino PM, Moreno CS, Varma V, Dong JT, Zhou W: Sox7 Is an independent checkpoint for beta-catenin function in prostate and colon epithelial cells. Mol Cancer Res 2008, 6:1421-1430.

25. Tsao CM, Yan MD, Shih YL, Yu PN, Kuo CC, Lin WC, Li HJ, Lin YW: SOX functions as a tumor suppressor by antagonizing the WNT/beta-catenin signaling pathway in hepatocellular carcinoma. Hepatology 2012, 56:2277-2287

26. Yan M, Zhang Y, He B, Xiang J, Wang Z-f, Zheng F-m, Xu J, Chen M-y, Zhu Y-I, Wen H-j, Wan X-b, Yue C-f, Yang N, Zhang W, Zhang J-I, Wang J, Wang Y, Li L-h, Zeng Y-x, Lam EWF, Hung M-C, Liu Q: IKKa restoration via EZH2 suppression induces nasopharyngeal carcinoma differentiation. Nat Commun 2014, 5:3661.

27. Watt FM: Involucrin and other markers of keratinocyte terminal differentiation. J Invest Dermatol 1983, 81:100s-103s.

28. Banks-Schlegel S, Green H: Involucrin synthesis and tissue assembly by keratinocytes in natural and cultured human epithelia. J Cell Biol 1981 90:732-737

29. Stosiek $P$, Kasper M, Moll R: Changes in cytokeratin expression accompany squamous metaplasia of the human respiratory epithelium. Virchows Arch A Pathol Anat Histopathol 1992, 421:133-141.

30. van Dorst EB, van Muijen GN, Litvinov SV, Fleuren GJ: The limited difference between keratin patterns of squamous cell carcinomas and adenocarcinomas is explicable by both cell lineage and state of differentiation of tumour cells. J Clin Pathol 1998, 51:679-684.

31. Zhang S, Li S, Gao JL: Promoter methylation status of the tumor suppressor gene SOX11 is associated with cell growth and invasion in nasopharyngeal carcinoma. Cancer Cell Int 2013, 13:109.

32. Zorn AM, Barish GD, Williams BO, Lavender P, Klymkowsky MW, Varmus HE: Regulation of Wnt signaling by Sox proteins: XSox17 alpha/beta and XSox3 physically interact with beta-catenin. Mol Cell 1999, 4:487-498.

33. Lin YW, Tsao CM, Yu PN, Shih YL, Lin CH, Yan MD: SOX1 suppresses cell growth and invasion in cervical cancer. Gynecol Oncol 2013, 131:174-181.

34. MacDonald BT, Tamai $\mathrm{K}$, He X: Wnt/beta-catenin signaling: components, mechanisms, and diseases. Dev Cell 2009, 17:9-26.

35. Clevers H, Nusse R: Wnt/beta-catenin signaling and disease. Cell 2012 , 149:1192-1205.

36. Kan L, Israsena N, Zhang Z, Hu M, Zhao LR, Jalali A, Sahni V, Kessler JA: Sox 1 acts through multiple independent pathways to promote neurogenesis. Dev Biol 2004, 269:580-594.

37. Buescher M, Hing FS, Chia W: Formation of neuroblasts in the embryonic central nervous system of Drosophila melanogaster is controlled by SoxNeuro. Development 2002, 129:4193-4203.

38. Lai HC, Lin MW, Huang TH, Yan P, Huang RL, Wang HC, Liu J, Chan MW, Chu TY, Sun CA, Chang CC, Yu MH: Identification of novel DNA methylation markers in cervical cancer. Int J Cancer 2008, 123:161-167.

39. Guan Z, Wang XR, Zhu XF, Huang XF, Xu J, Wang LH, Wan XB, Long ZJ, Liu JN, Feng GK, Huang W, Zeng YX, Chen FJ, Liu Q: Aurora-A, a negative prognostic marker, increases migration and decreases radio sensitivity in cancer cells. Cancer Res 2007, 67:10436-10444.

40. Wang LH, Xiang J, Yan M, Zhang Y, Zhao Y, Yue CF, Xu J, Zheng FM, Chen JN, Kang Z, Chen TS, Xing D, Liu Q: The mitotic kinase Aurora-A induces mammary cell migration and breast cancer metastasis by activating the Cofilin-F-actin pathway. Cancer Res 2010, 70:9118-9128.

41. Xu J, Wu X, Zhou WH, Liu AW, Wu JB, Deng JY, Yue CF, Yang SB, Wang J, Yuan ZY, Liu Q: Aurora-A identifies early recurrence and poor prognosis and promises a potential therapeutic target in triple negative breast cancer. PLoS One 2013, 8:e56919.

42. Dontu G, Abdallah WM, Foley JM, Jackson KW, Clarke MF, Kawamura MJ, Wicha MS: In vitro propagation and transcriptional profiling of human mammary stem/progenitor cells. Genes Dev 2003, 17:1253-1270.

43. Bouska A, Lushnikova T, Plaza S, Eischen CM: Mdm2 promotes genetic instability and transformation independent of p53. Mol Cell Biol 2008, 28:4862-4874

doi:10.1186/1476-4598-13-257

Cite this article as: Guan et al:: SOX1 down-regulates $\beta$-catenin and reverses malignant phenotype in nasopharyngeal carcinoma. Molecular Cancer 2014 13:257. 